Original paper

\title{
Petrology of the Late Cretaceous peralkaline rhyolites (pantellerite and comendite) from Lake Chad, Central Africa
}

\author{
Gbambie Isaac Bertrand MBOWOU ${ }^{*}$, Claudial LAGMET², Sébastien NOMADE ${ }^{3}$, Ismaïla \\ NGOUNOUNO ${ }^{4}$, Bernard DÉRUELLE ${ }^{5}$, Daniel OHNENSTETTER ${ }^{6}$
}

${ }^{1}$ École de Géologie et d'Exploitation Minière (EGEM), Université de Ngaoundéré, BP: 454 Ngaoundéré, Cameroun; mbowou2000@yahoo.fr

2 Institut Universitaire Polytechnique de Mongo, BP: 4377 N’Djaména, Tchad

${ }^{3}$ Laboratoire des Sciences du Climat et de l'Environnement, IPSL, CEA-CNRS-UVSQ, Avenue de la Terrasse, 91198 Gif-sur-Yvette Cedex, France

${ }^{4}$ Faculté des Sciences, Université de Ngaoundéré, BP: 454 Ngaoundéré, Cameroun

${ }^{5}$ Université Pierre-et-Marie-Curie et IUFM Académie de Versailles, 4, place Jussieu, 75252 Paris cedex 05, France

${ }^{6}$ Centre de recherches pétrographiques et géochimiques, UFR A9046, 15, rue Notre-Dame-des-Pauvres, B.P. 20, 54501 Vandoeuvre-les-Nancy cedex, France

* Corresponding author

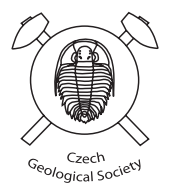

Late Cretaceous peralkaline rhyolites $\left(69.4 \pm 0.4 \mathrm{Ma}-2 \sigma\right.$ analytical, $1.9 \mathrm{Ma}$ full external uncertainty, ${ }^{40} \mathrm{Ar} /{ }^{39} \mathrm{Ar}$ sanidine single crystal laser dating) from Lake Chad are the oldest lavas of the "Cameroon Hot Line". These lavas (pantellerite and comendite), consisting of quartz \pm alkali feldspar \pm fluoro-arfvedsonite \pm augite-hedenbergite \pm aegirine \pm fayalite $\left(\mathrm{Fa}_{97-99}\right)$ and ilmenite, are characterized by a progressive increase in the total REE contents and the magnitude of the negative Eu anomaly owing to the alkali feldspar-dominated fractionation. Two groups of peralkaline rhyolites have been distinguished according to mineralogical and geochemical data. Both, group-1 $\left(\mathrm{La}_{\mathrm{N}} / \mathrm{Yb}_{\mathrm{N}}: 8.4-9.0 ; \mathrm{Zr} / \mathrm{Nb}: \sim 8\right)$ and group-2 $\left(\mathrm{La}_{\mathrm{N}} / \mathrm{Yb}_{\mathrm{N}}: 10.7-12.6 ; \mathrm{Zr} / \mathrm{Nb}: \sim 6\right)$ are genetically related. The peralkaline rhyolites from Lake Chad could not be solely the product of fractional crystallization from a basaltic parental magma. The influx of F-rich fluids could have greatly modified the composition of rhyolitic magmas as attested by the presence of fluoro-arfvedsonite.

Keywords: petrology, geochemistry, Cretaceous, peralkaline rhyolites, Lake Chad, Cameroon Line

Received: 18 March 2012; accepted: 5 June 2012; handling editor: V. Kachlik

The online version of this article (doi: 10.3190/jgeosci.118) contains supplementary electronic material.

\section{Introduction}

Peralkaline rocks are commonly emplaced in both tectonomagmatic oceanic intraplate and continental domains such as in Ethiopia (Peccerillo et al. 2003, 2007; Ronga et al. 2010), Kenya (Weaver et al. 1972; Baker and Henage 1977; Scaillet and Macdonald 2001, 2003; Heumann and Davies 2002; Marshall et al. 2009; Macdonald and Bagiński 2009), SW Sardinia (Morra et al. 1994) and Pantelleria Island, Italy (Lowenstern and Mahood 1991; Civetta et al. 1998; Avanzinelli et al. 2004). An excellent example of such a volcanic association is provided by the rhyolites occurring along the "Cameroon Hot Line", which were studied in its continental sector (Mbépit Massif, Kapsiki Plateau, Garoua Trough, Benue Valley, Lake Chad, Bambouto; Ngounouno et al. 1997, 2000, 2003; Marzoli et al. 1999; Vicat et al. 2002; Wandji et al. 2008).

In this paper, new mineralogical, geochronological and geochemical data for peralkaline rhyolites from Lake Chad will be presented and compared to other rhyolite occurrences from the "Cameroon Hot Line". These data are used to constrain the nature, age and genesis of evolved lavas of the Lake Chad area.

\section{Geological setting}

The Lake Chad Basin is composed of both Late Cretaceous volcanic and Cenozoic to Quaternary sedimentary formations (Vicat et al. 2002; Ganwa et al. 2009), underlain by a Precambrian basement consisting of gneiss and migmatites (Schroeter and Gear 1973). According to the exploratory drilling at Logone-Birni and Bol (Lake Chad Basin), this basement is located at about $600 \mathrm{~m}$ depth (Barbeau 1956). The volcanic eruptions were probably triggered after the reactivation of Precambrian basement faults (Moreau et al. 1987) that could have been linked to the occurrence of hot lines in the asthenospheric mantle (Bonatti et al. 1976). Lake Chad Basin was an active tectonic pull-apart basin (a graben, according to Vicat et al. 


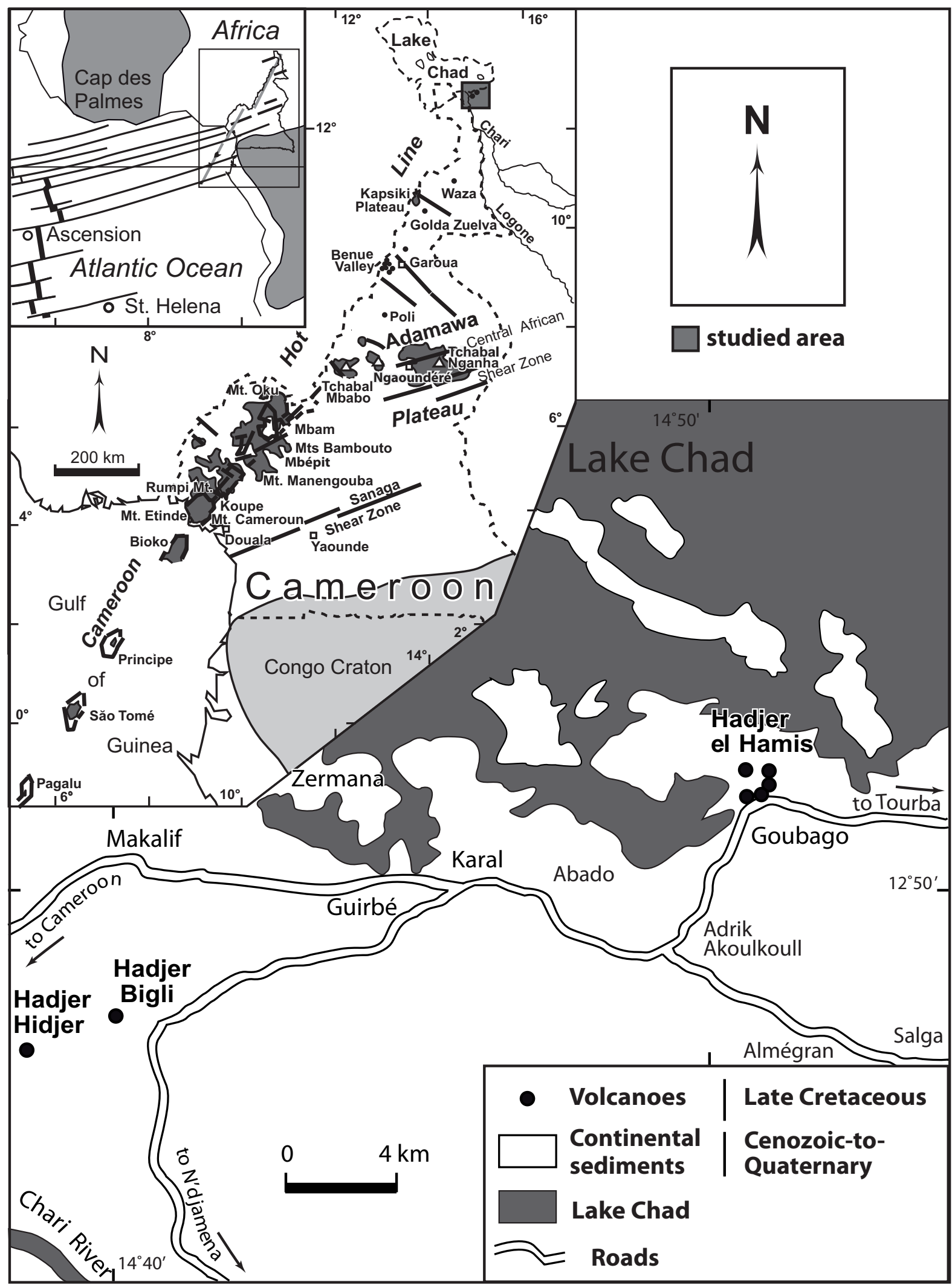

Fig. 1 Location of the studied area, tectono-magmatic settings of the Cameroon Hot Line (Déruelle et al. 2007) and sampling sites of peralkaline rhyolites from Lake Chad. 
2002), located on the northern Congo Craton (Fig. 1). The tectonic plates that affected Africa and South America during the Aptian and Albian times, leading to the opening of the Central Atlantic Ocean (Benkhelil 1986), contributed to the formation in the Lake Chad Basin (Louis 1970) of Agadem and Bornu troughs (Vicat et al. 2002) and the associated volcanism.

Peralkaline rhyolites from SE of Lake Chad (Fig. 1) were sampled on rocky slopes and/or in working quarries at the flanks of Hadjer el Hamis, Hadjer Bigli and Hadjer Hidjer volcanoes, at the northeastern end of the Cameroon Line (Déruelle et al. 1991). The Cameroon Line, recently named "Cameroon Hot Line", is an active intraplate tectonomagmatic zone, with associated alkaline volcanism, developed on both oceanic and continental lithosphere. Oriented $\mathrm{N} 30^{\circ} \mathrm{E}$, it crosses the Gulf of Guinea and Cameroon for more than $2000 \mathrm{~km}$, from Pagalu Island to Lake Chad (Déruelle et al. 2007).

Hadjer el Hamis volcanoes (418 m a.s.l.) are five necks composed of dark green rhyolites, showing vertical columnar jointing. The rhyolitic fragments split from the flanks are scattered around the foothills. The Hadjer Bigli plug (308 $\mathrm{m}$ a.s.l.) is made up of strongly weathered reddish brown rhyolite, which is cut by greenish dark rhyolitic dykes. This volcano of c. 15-20 m height is slightly flattened on its top. Hadjer Hidjer (283 m a.s.1.) is a flattened and slightly elongated $\left(\mathrm{N} 30^{\circ} \mathrm{E}\right)$ plug-dome surrounded by continental sedimentary rocks, which is made up of greenish dark rhyolite belonging to the inner core of the small lava plug. Its surface is covered by lava blocks, broken along columnar and tabular joints, which were likely produced by the internal motion of the highly viscous magma.

\section{Analytical methods}

Mineral phases (olivine, clinopyroxene, amphibole, alkali feldspars, Fe-Ti oxides, quartz) have been analyzed by electron microprobes CAMEBAX SX50 and SX100 at Université Pierre-et-Marie Curie, Paris. The measurements were made according to standard analyzed data, under the conditions expressed in $\mathrm{kV}$ (accelerating voltage), nA (beam current) and s (counting times at the peak). Olivine $(15 \mathrm{kV}, 40 \mathrm{nA}, 20 \mathrm{~s}$ for all elements, except Si (10 s)), clinopyroxene (15 kV, $40 \mathrm{nA}, 20 \mathrm{~s}$ for $\mathrm{Si}, \mathrm{Al}, \mathrm{Fe}, \mathrm{Mg}, \mathrm{Ca}, \mathrm{Na}, \mathrm{Mn}$ and $30 \mathrm{~s}$ for $\mathrm{Ti}$ and $\mathrm{Zr}$ ), amphibole $(15 \mathrm{kV}, 10 \mathrm{nA}, 15 \mathrm{~s}$ for $\mathrm{Si}, \mathrm{Al}, \mathrm{Mg}, \mathrm{Na}$ and $\mathrm{K}$, $20 \mathrm{~s}$ for $\mathrm{Ca}$ and $\mathrm{Ti}, 25 \mathrm{~s}$ for $\mathrm{Fe}$ and $\mathrm{Mn}, 30 \mathrm{~s}$ for $\mathrm{Cl}$ and F), feldspar (15 kV, $10 \mathrm{nA}, 5 \mathrm{~s}$ for all elements), $\mathrm{Fe}-\mathrm{Ti}$ oxides $(15 \mathrm{kV}, 40 \mathrm{nA}, 40 \mathrm{~s}$ for Ti, Fe, Mn, Mg; $10 \mathrm{~s}$ for $\mathrm{Si}, 15 \mathrm{~s}$ for $\mathrm{Cr}$ and $30 \mathrm{~s}$ for $\mathrm{Al})$. Measurements correction was carried out using the "PAP" program (Pouchou and Pichoir 1991). Analyses are given in terms of oxides of the elements (wt. \%). The recalculations of amphibole formulae were undertaken using the script of Tindle and Webb (1994).

Whole-rock chemical analyses of peralkaline rhyolites from Lake Chad were carried out at CRPG laboratory, Nancy. Major elements were analyzed by ICP-AES and trace elements by ICP-MS. The samples were previously selected in order to limit superficial contamination, then crushed. Details of other analytical processes were presented elsewhere (Carignan et al. 2001).

The dating of a peralkaline rhyolite sample from Lake Chad (Hadjer el Hamis, Tab. 1) was performed by ${ }^{40} \mathrm{Ar} /{ }^{39} \mathrm{Ar}$ method at Laboratoire des Sciences du Climat et de l'Environnement, CEA-CNRS-UVSQ, France. For ${ }^{40} \mathrm{Ar} /{ }^{39} \mathrm{Ar}$ dating, pristine sanidine crystals $200-250 \mu \mathrm{m}$ in size were handpicked under a binocular and slightly leached for 5 minutes in a $5 \% \mathrm{HF}$ acid solution. A total of 15 single crystals were finally handpicked after leaching and separately loaded in a single pit in an aluminum disk. Sample was irradiated for 90 minutes (Irr 14) in the $\beta_{1}$ tube of the OSIRIS reactor (CEA Saclay, France). After irradiation, crystals were transferred one by one into a copper sample holder and then loaded into a differential vacuum Cleartran ${ }^{\odot}$ window. A total of 10 crystals were individually fused at about $12 \%$ of the full laser power. Argon isotopes were analyzed using a VG 5400 mass spectrometer equipped with a single ion counter $\left(\right.$ Balzers $^{\mathcal{O}}$ SEV 217 SEN) following procedures outlined in Nomade et al. (2010). Each Ar isotope measurement consisted of 20 cycles of peak switching of the argon isotopes. Neu-

Tab. 1 Overview of geochronological data for rhyolites from the "Cameroon Hot Line"

\begin{tabular}{lcccc}
\hline location & lavas & analytical methods & ages (Ma) & references \\
\hline Mbépit Massif & rhyolite & $\mathrm{K}-\mathrm{Ar}$ & $45.5 \pm 1.1$ & Wandji et al. (2008) \\
& rhyolite & $\mathrm{K}-\mathrm{Ar}$ & $44.0 \pm 1.0$ & Wandji et al. (2008) \\
Mts Bambouto & rhyolite & $\mathrm{K}-\mathrm{Ar}$ & 16.0 & Marzoli et al. (1999) \\
Mt. Oku & rhyolite & $\mathrm{K}-\mathrm{Ar}$ & 23.2 & Dunlop (1983) \\
Benue valley & ignimbritic rhyolite & $\mathrm{K}-\mathrm{Ar}$ & $24.8 \pm 0.1$ & Dunlop (1983) \\
Kapsiki Plateau & rhyolite & $\mathrm{K}-\mathrm{Ar}$ & $36.8 \pm 0.9$ & Ngounouno et al. (2003) \\
& rhyolite & $\mathrm{Rb}-\mathrm{Sr}$ & $29 \pm 0.5$ & Dunlop (1983) \\
Lake Chad & rhyolite & $\mathrm{Kb}-\mathrm{Sr}$ & $32 \pm 0.5$ & Dunlop (1983) \\
& peralkaline rhyolite & $\mathrm{Ar}-\mathrm{Ar}$ & $68.9 \pm 1.4$ & Schroeter and Gear (1973) \\
\hline
\end{tabular}


tron fluence $(\mathrm{J})$ was monitored by co-irradiation of Alder Creek sanidine (ACs, Nomade et al. 2005) placed in the same pit as the leucite. The J value was determined from analyses of three single ACs crystals. Corresponding $\mathrm{J}$ value (see online electronic supplement, Appendix 1, for full data table) was calculated using an age of 1.193 $\mathrm{Ma}$ (Nomade et al. 2005) and the total decay constant of Steiger and Jäger (1977). The precision and accuracy of the mass discrimination correction was monitored by daily measurements of air argon (see full experimental description in Nomade et al. 2010).

\section{Petrography}

\subsection{Hadjer Bigli (FB)}

The main body of the Hadjer Bigli plug is composed of reddish brown rhyolites, with partly welded tuff appearance. These rhyolites display quartz and alkali feldspar phenocrysts set in an altered devitrified glassy groundmass, indicating late to post-magmatic alteration. Alkali feldspar is euhedral to subhedral, present as both phenocrysts and groundmass phase. Some alkali feldspar phenocrysts are altered and crossed by brown bands. Quartz occurs as euhedral phenocrysts (2.1-1.2 $\mathrm{mm})$. The greenish dark rhyolite dykes from Hadjer Bigli are microlithic porphyritic, with quartz (1-2 mm), alkali feldspars $(\sim 1.5 \mathrm{~mm})$, green amphibole $(0.4-1.5$ $\mathrm{mm})$, clinopyroxene $(\sim 0.5 \mathrm{~mm})$ and Fe-Ti oxide $(\sim 0.9$ $\mathrm{mm}$ ) phenocrysts, in a groundmass consisting of alkali feldspar, amphibole, clinopyroxene and Fe-Ti oxide microlites, unevenly distributed in the glass. The quartz phenocrysts are sometimes agglomerated and $\mathrm{Fe}-\mathrm{Ti}$ oxide inclusions are present in the alkali feldspar phenocrysts.

\subsection{Hadjer el Hamis (DE)}

Rhyolitic samples from Hadjer el Hamis are porphyritic and partly brecciated. They contain phenocrysts of quartz (up to $2.5 \mathrm{~mm}$ ), alkali feldspar $(\sim 3.5 \mathrm{~mm})$, amphibole, clinopyroxene and $\mathrm{Fe}-\mathrm{Ti}$ oxide, in a groundmass consisting of alkali feldspar, greenish amphibole and $\mathrm{Fe}-\mathrm{Ti}$ oxides microlites enclosed by a thin glassy phase. Quartz phenocrysts are euhedral, with corrosion gulfs. Clinopyroxene phenocrysts are overgrown and partly replaced by Fe-Ti oxides. Several small $(1.5 \mathrm{~cm})$ basaltic xenoliths occur in these samples. They are strongly altered and carry scarce phenocrysts of mafic minerals (e.g., olivine and clinopyroxene) and clear plagioclase which are scattered in the dark-brownish glassy groundmass. The mafic phenocrysts are sometimes pseudomorphosed by fine-grained $\mathrm{Fe}-\mathrm{Ti}$ oxides.

\subsection{Hadjer Hidjer (DH)}

The porphyritic rhyolite from Hadjer Hidjer is partly brecciated and contains xenoliths of basaltic fragments. Quartz (1.3 mm), alkali feldspar (3 $\mathrm{mm})$, clinopyroxene, amphibole $(1.8 \mathrm{~mm}), \mathrm{Fe}-\mathrm{Ti}$ oxide $(0.5 \mathrm{~mm})$ phenocrysts and xenoliths of basalt (see Ganwa et al. 2009) are enclosed in a groundmass of amphibole, clinopyroxene and $\mathrm{Fe}-\mathrm{Ti}$ oxides microlites. The pleochroic amphibole phenocrysts are scattered and sometimes show olivine inclusions enclosed in a reddish brown core, frequently altered to $\mathrm{Fe}-\mathrm{Ti}$ oxides.

\section{Mineral chemistry data}

Electron microprobe compositions of representative mineral phases of rhyolites from Lake Chad are reported in Tabs 2-6. Compositional variation of pyroxene, amphibole and feldspar are illustrated in Figs $2 \mathrm{a}-\mathrm{b}$ to 3 .

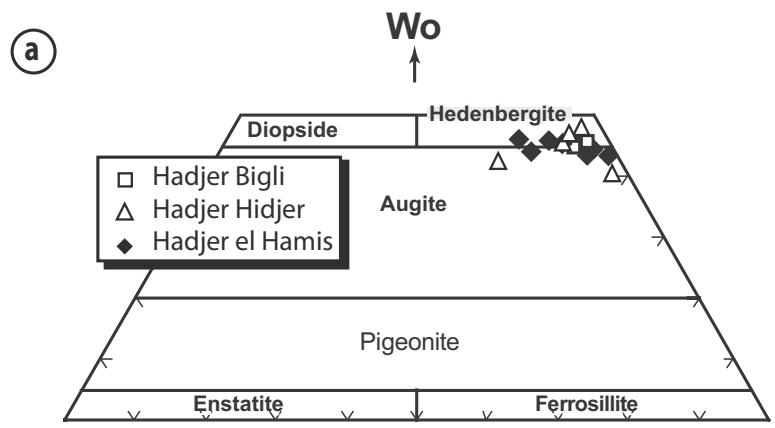

En

Fs

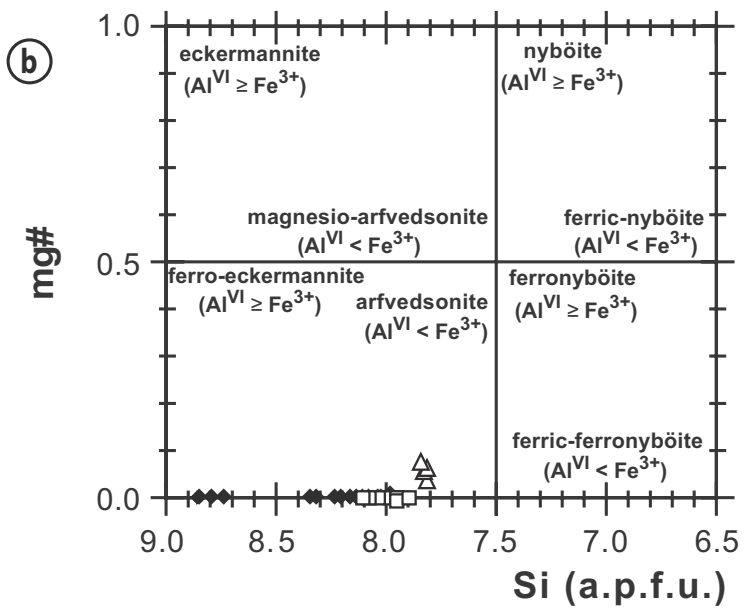

Fig. 2 Compositional variations of ferromagnesian minerals from the Lake Chad rhyolites: a - classification of clinopyroxene in the Wo-EnFs ternary diagram (Morimoto 1989); b - classification diagram mg\# vs Si (according to Leake et al. 1997) for amphibole. 
Tab. 2 Selected compositions of olivine from peralkaline rhyolites of Lake Chad (wt. \% and a.p.f.u. on the basis of 4 oxygens)

\begin{tabular}{lccc}
\hline Sample & $\mathrm{DH} 3$ & $\mathrm{DH}$ & $\mathrm{DE}$ \\
description & $\mathrm{ph}$ & $\mathrm{ph}$ & $\mathrm{ph}$ \\
\hline $\mathrm{SiO}_{2}$ (wt. \%) & 30.41 & 39.24 & 38.65 \\
$\mathrm{Al}_{2} \mathrm{O}_{3}$ & 0.00 & 0.09 & 0.01 \\
$\mathrm{FeO}$ & 64.95 & 56.36 & 55.39 \\
$\mathrm{MnO}$ & 3.00 & 2.16 & 3.90 \\
$\mathrm{MgO}$ & 0.95 & 0.35 & 0.34 \\
$\mathrm{CaO}$ & 0.44 & 0.76 & 0.66 \\
$\mathrm{Sum}$ & 99.75 & 98.96 & 98.95 \\
\hline $\mathrm{Si} \mathrm{(a.p.f.u.)}$ & 1.017 & 1.217 & 1.206 \\
$\mathrm{Al}$ & 0.000 & 0.003 & 0.000 \\
$\mathrm{Fe}$ & + & 1.462 & 1.446 \\
$\mathrm{Mn}$ & 1.817 & 0.057 & 0.103 \\
$\mathrm{Mg}$ & 0.085 & 0.016 & 0.016 \\
$\mathrm{Ca}$ & 0.047 & 0.025 & 0.022 \\
\hline $\mathrm{Fo} \mathrm{(mol.} \mathrm{\% )}$ & 0.016 & 1.06 & 1.01 \\
$\mathrm{Fa}$ & 2.44 & 98.95 & 98.99 \\
\hline
\end{tabular}

ph: phenocrysts; a.p.f.u.: atom per formula unit

\subsection{Olivine}

Fayalite-rich olivine $\left(\mathrm{Fa}_{97-99}\right)$ was analyzed in rhyolites from Hadjer el Hamis and Hadjer Hidjer (Tab. 2). The $\mathrm{CaO}$ contents are high (up to $0.76 \mathrm{wt}$. \%), indicating low crystallization pressures near the surface (Simkin and Smith $1970)$, at low temperatures $\left(<900{ }^{\circ} \mathrm{C}\right.$, estimated after Roeder and Emslie 1970). Fayalite phenocrysts are partly or widely replaced by fine-grained aggregates of $\mathrm{Fe}$-Ti oxides.

\subsection{Clinopyroxene}

Clinopyroxene (Tab. 3) analyses straddle the boundary of the hedenbergite and augite fields (Fig. 2a). The $\mathrm{TiO}_{2}$ contents range from 0.35 to 1.00 wt. \%. At Hadjer Hidjer occur rare phenocrysts of aegirine-augite, characterized by high $\mathrm{TiO}_{2}$ contents $(7.3 \mathrm{wt}$. \%) together with augitehedenbergite. On the other hand, at Hadjer Bigli were recorded aegirine phenocrysts with $0.89 \mathrm{wt} \% \mathrm{TiO}_{2}$. High $\mathrm{TiO}_{2}$ contents in sodic clinopyroxene indicate low crystal-

Tab. 3 Clinopyroxene compositions (wt. \% and a.p.f.u. on the basis of 6 oxygens)

\begin{tabular}{|c|c|c|c|c|c|c|c|c|c|c|c|c|c|}
\hline $\begin{array}{l}\text { Sample } \\
\text { description }\end{array}$ & $\begin{array}{c}\mathrm{DH} 2 \\
\text { ph }\end{array}$ & $\begin{array}{l}\mathrm{DH} 3 \\
\mathrm{ph}, \mathrm{c}\end{array}$ & $\begin{array}{l}\mathrm{DH} 3 \\
\mathrm{ph}, \mathrm{c}\end{array}$ & $\begin{array}{c}\text { DH4 } \\
\text { ph,r }\end{array}$ & $\begin{array}{l}\mathrm{DH} 4 \\
\mathrm{ph}, \mathrm{r}\end{array}$ & $\begin{array}{l}\text { DH4 } \\
\text { ph,c }\end{array}$ & $\begin{array}{l}\mathrm{DE} \\
\text { ph,c }\end{array}$ & $\begin{array}{l}\text { DE } \\
\text { ph,c }\end{array}$ & $\begin{array}{l}\mathrm{DE} \\
\mathrm{ph}, \mathrm{c}\end{array}$ & $\begin{array}{l}\mathrm{DE} \\
\mathrm{ph}, \mathrm{r}\end{array}$ & $\begin{array}{l}\mathrm{DE} \\
\text { ph,c }\end{array}$ & $\begin{array}{c}\text { DH4 } \\
\text { ph }\end{array}$ & $\begin{array}{c}\text { FB1 } \\
\text { ph }\end{array}$ \\
\hline $\mathrm{SiO}_{2}($ wt. \%) & 49.20 & 48.37 & 48.98 & 49.61 & 49.67 & 48.52 & 49.34 & 48.43 & 46.93 & 48.21 & 48.71 & 41.56 & 53.1 \\
\hline $\mathrm{TiO}_{2}$ & 0.43 & 0.39 & 0.42 & 0.37 & 0.24 & 0.40 & 0.63 & 0.32 & 1.00 & 0.35 & 0.38 & 7.34 & 0.89 \\
\hline $\mathrm{Al}_{2} \mathrm{O}_{3}$ & 0.29 & 0.17 & 0.16 & 0.16 & 0.19 & 0.47 & 0.68 & 0.11 & 1.00 & 0.15 & 0.16 & 0.06 & 0.15 \\
\hline $\mathrm{FeO}$ & 26.48 & 28.38 & 28.46 & 29.11 & 28.66 & 26.2 & 22.64 & 28.94 & 26.04 & 28.67 & 28.99 & 40.69 & 30.56 \\
\hline $\mathrm{MnO}$ & 1.06 & 1.27 & 1.19 & 1.20 & 0.99 & 1.14 & 1.36 & 1.02 & 1.28 & 1.01 & 1.07 & 1.42 & 0.30 \\
\hline $\mathrm{CaO}$ & 18.96 & 18.36 & 17.82 & 15.51 & 15.99 & 18.65 & 19.14 & 16.42 & 20.03 & 18.33 & 17.12 & 0.15 & 0.37 \\
\hline $\mathrm{Na}_{2} \mathrm{O}$ & 1.26 & 1.41 & 1.39 & 2.33 & 2.95 & 1.06 & 0.47 & 2.22 & 0.50 & 2.02 & 1.78 & 7.14 & 14.31 \\
\hline $\mathrm{ZrO}_{2}$ & & & 0.03 & & & & & & 0.06 & 0.06 & & & \\
\hline Sum & 99.15 & 99.12 & 99.18 & 99.30 & 99.74 & 99.73 & 99.47 & 98.55 & 99.16 & 99.51 & 99.33 & 98.50 & 99.68 \\
\hline $\mathrm{Fe}_{2} \mathrm{O}_{3}$ (calc.) & 1.20 & 3.23 & 1.43 & 3.49 & 6.76 & 4.05 & 0.48 & 5.60 & 2.70 & 6.49 & 4.07 & 21.53 & 37.11 \\
\hline FeO (calc.) & 25.40 & 25.48 & 27.17 & 25.97 & 22.58 & 22.56 & 22.20 & 23.90 & 23.61 & 22.83 & 25.33 & 21.32 & 2.83 \\
\hline Si (a.p.f.u.) & 2.011 & 1.990 & 2.016 & 2.023 & 2.001 & 1.957 & 1.976 & 1.989 & 1.923 & 1.965 & 1.992 & 1.721 & 1.969 \\
\hline $\mathrm{Ti}$ & 0.013 & 0.012 & 0.013 & 0.011 & 0.007 & 0.012 & 0.019 & 0.009 & 0.031 & 0.011 & 0.012 & 0.229 & 0.025 \\
\hline $\mathrm{Al}$ & 0.014 & 0.008 & 0.008 & 0.008 & 0.009 & 0.022 & 0.032 & 0.005 & 0.048 & 0.007 & 0.008 & 0.003 & 0.007 \\
\hline $\mathrm{Fe}^{3+}$ & 0.037 & 0.099 & 0.044 & 0.107 & 0.205 & 0.123 & 0.015 & 0.173 & 0.083 & 0.199 & 0.125 & 0.671 & 1.035 \\
\hline $\mathrm{Fe}^{2+}$ & 0.868 & 0.877 & 0.935 & 0.886 & 0.761 & 0.761 & 0.744 & 0.821 & 0.809 & 0.778 & 0.866 & 0.738 & 0.000 \\
\hline $\mathrm{Mn}$ & 0.037 & 0.044 & 0.042 & 0.042 & 0.034 & 0.039 & 0.046 & 0.036 & 0.044 & 0.035 & 0.037 & 0.049 & 0.009 \\
\hline $\mathrm{Mg}$ & 0.089 & 0.047 & 0.045 & 0.061 & 0.063 & 0.198 & 0.311 & 0.067 & 0.142 & 0.043 & 0.068 & 0.009 & 0,000 \\
\hline $\mathrm{Ca}$ & 0.830 & 0.809 & 0.786 & 0.678 & 0.690 & 0.806 & 0.821 & 0.723 & 0.879 & 0.801 & 0.750 & 0.007 & 0.015 \\
\hline $\mathrm{Na}$ & 0.099 & 0.113 & 0.111 & 0.184 & 0.230 & 0.083 & 0.037 & 0.177 & 0.040 & 0.159 & 0.141 & 0.573 & 1.029 \\
\hline $\mathrm{Zr}$ & & & 0.001 & & & & & & 0.001 & 0.001 & & & \\
\hline $\mathrm{Fe}^{3+} / \mathrm{Fe}^{2+}$ & 0.043 & 0.113 & 0.047 & 0.121 & 0.269 & 0.162 & 0.020 & 0.211 & 0.103 & 0.256 & 0.144 & 0.909 & \\
\hline Wo (mol. \%) & 45.43 & 45.30 & 43.20 & 40.03 & 44.45 & 43.68 & 42.20 & 43.60 & 45.64 & 47.77 & 43.28 & & \\
\hline En & 4.17 & 2.80 & 1.30 & 2.11 & 3.97 & 11.75 & 17.05 & 4.24 & 8.20 & 2.78 & 4.15 & & \\
\hline $\mathrm{J}$ & 0.20 & 0.23 & 0.22 & 0.37 & 0.46 & 0.17 & 0.07 & 0.35 & 0.08 & 0.32 & 0.28 & 1.15 & 2.06 \\
\hline
\end{tabular}

c: core ; r: rim; DH: Hadjer Hidjer; DE: Hadjer el Hamis; FB: Hadjer Bigli 
Tab. 4 Amphibole compositions (wt. \% and a.p.f.u. on the basis of 23 oxygens)

\begin{tabular}{|c|c|c|c|c|c|c|c|c|c|}
\hline $\begin{array}{l}\text { Sample } \\
\text { description }\end{array}$ & $\begin{array}{l}\mathrm{DH} 3 \\
\mathrm{ph}, \mathrm{c}\end{array}$ & $\begin{array}{l}\text { DH3 } \\
\text { ph,r }\end{array}$ & $\begin{array}{l}\text { DH4 } \\
\text { ph,r }\end{array}$ & $\begin{array}{c}\mathrm{DE} \\
\mathrm{ph}, \mathrm{c}\end{array}$ & $\begin{array}{c}\mathrm{DE} \\
\mathrm{ph}, \mathrm{c}\end{array}$ & $\begin{array}{l}\text { FB1 } \\
\text { ph,c }\end{array}$ & $\begin{array}{l}\text { FB1 } \\
\text { ph,c }\end{array}$ & $\begin{array}{l}\text { FB1 } \\
\text { ph,c }\end{array}$ & $\begin{array}{l}\text { FB1 } \\
\text { ph,c }\end{array}$ \\
\hline $\mathrm{SiO}_{2}$ (wt. \%) & 48.97 & 49.51 & 49.98 & 50.83 & 50.31 & 50.65 & 49.96 & 49.81 & 49.01 \\
\hline $\mathrm{TiO}_{2}$ & 1.15 & 0.93 & 0.18 & 1.04 & 0.75 & 0.75 & 0.69 & 0.81 & 1.15 \\
\hline $\mathrm{Al}_{2} \mathrm{O}_{3}$ & 0.77 & 0.37 & 0.28 & 0.35 & 0.40 & 0.34 & 0.31 & 0.33 & 0.74 \\
\hline $\mathrm{FeO}$ & 32.15 & 33.69 & 33.69 & 33.96 & 34.67 & 34.94 & 34.17 & 34.27 & 33.83 \\
\hline $\mathrm{MnO}$ & 1.01 & 1.10 & 1.12 & 0.61 & 0.68 & 0.72 & 0.68 & 0.73 & 0.75 \\
\hline $\mathrm{MgO}$ & 0.97 & 0.80 & 1.15 & 0.03 & 0.02 & 0.00 & 0.00 & 0.01 & 0.13 \\
\hline $\mathrm{CaO}$ & 2.95 & 3.01 & 2.88 & 0.74 & 1.02 & 0.70 & 0.60 & 0.95 & 2.52 \\
\hline $\mathrm{Na}_{2} \mathrm{O}$ & 6.75 & 6.90 & 6.71 & 8.04 & 7.84 & 8.34 & 8.09 & 8.13 & 7.35 \\
\hline $\mathrm{K}_{2} \mathrm{O}$ & 1.46 & 1.38 & 1.37 & 1.82 & 1.52 & 1.45 & 1.62 & 1.37 & 1.36 \\
\hline $\mathrm{F}$ & 2.45 & 2.54 & 2.85 & 1.78 & 2.06 & 2.33 & 2.47 & 1.93 & 2.16 \\
\hline $\mathrm{Cl}$ & 0.06 & 0.05 & 0.05 & 0.01 & 0.01 & 0.01 & 0.01 & 0.00 & 0.02 \\
\hline $\mathrm{F}$ as $\mathrm{O}$ & 1.03 & 1.07 & 1.20 & 0.75 & 0.87 & 0.98 & 1.04 & 0.81 & 0.91 \\
\hline $\mathrm{Cl}$ as $\mathrm{O}$ & 0.01 & 0.01 & 0.01 & 0.00 & 0.00 & 0.00 & 0.00 & 0.00 & 0.00 \\
\hline Sum & 99.73 & 101.36 & 101.47 & 99.96 & 100.15 & 101.21 & 99.64 & 99.15 & 99.93 \\
\hline $\mathrm{Si}$ (a.p.f.u) & 7.965 & 7.951 & 7.994 & 8.159 & 8.080 & 8.085 & 8.119 & 8.084 & 7.977 \\
\hline $\mathrm{Al}^{\mathrm{IV}}$ & 0.035 & 0.049 & 0.006 & 0.000 & 0.000 & 0.000 & 0.000 & 0.000 & 0.023 \\
\hline $\mathrm{Al}^{\mathrm{VI}}$ & 0.112 & 0.022 & 0.046 & 0.066 & 0.076 & 0.064 & 0.059 & 0.063 & 0.119 \\
\hline $\mathrm{Ti}$ & 0.141 & 0.112 & 0.022 & 0.126 & 0.091 & 0.090 & 0.084 & 0.099 & 0.141 \\
\hline $\mathrm{Fe}^{3+}$ & 0.182 & 0.335 & 0.569 & 0.235 & 0.480 & 0.471 & 0.440 & 0.399 & 0.141 \\
\hline $\mathrm{Fe}^{2+}$ & 4.191 & 4.190 & 3.937 & 4.324 & 4.177 & 4.193 & 4.203 & 4.252 & 4.464 \\
\hline $\mathrm{Mn}$ & 0.139 & 0.150 & 0.152 & 0.083 & 0.093 & 0.097 & 0.094 & 0.100 & 0.103 \\
\hline $\mathrm{Mg}$ & 0.235 & 0.192 & 0.274 & 0.007 & 0.005 & 0.000 & 0.000 & 0.002 & 0.032 \\
\hline $\mathrm{Ca}$ & 0.514 & 0.518 & 0.494 & 0.127 & 0.176 & 0.120 & 0.104 & 0.165 & 0.439 \\
\hline $\mathrm{Na}$ & 2.129 & 2.149 & 2.081 & 2.502 & 2.441 & 2.581 & 2.549 & 2.558 & 2.320 \\
\hline $\mathrm{K}$ & 0.303 & 0.283 & 0.280 & 0.373 & 0.311 & 0.295 & 0.336 & 0.284 & 0.282 \\
\hline $\mathrm{F}$ & 1.260 & 1.290 & 1.442 & 0.904 & 1.046 & 1.176 & 1.269 & 0.991 & 1.112 \\
\hline $\mathrm{Cl}$ & 0.017 & 0.014 & 0.014 & 0.003 & 0.003 & 0.003 & 0.003 & 0.000 & 0.006 \\
\hline $\mathrm{OH}$ & 0.723 & 0.696 & 0.545 & 1.094 & 0.951 & 0.821 & 0.728 & 1.009 & 0.883 \\
\hline $\mathrm{Mg} /\left(\mathrm{Mg}+\mathrm{Fe}^{2+}\right)$ & 0.053 & 0.044 & 0.065 & 0.002 & 0.001 & 0.000 & 0.000 & 0.001 & 0.007 \\
\hline
\end{tabular}

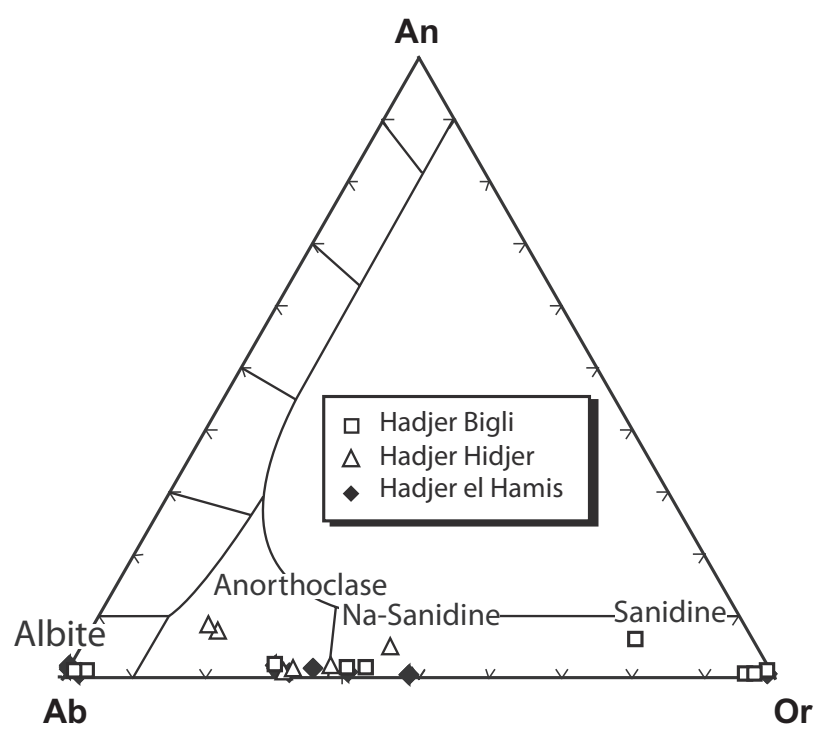

Fig. $3 \mathrm{An}-\mathrm{Ab}-\mathrm{Or}$ diagram for alkali feldspar of the peralkaline rhyolites from Lake Chad. lization temperature $\left(<600{ }^{\circ} \mathrm{C}\right.$, Ferguson 1977) as showed for the aegirine from the Djinga Tadorgal lavas (Mbowou et al. 2010). The crystallization of aegirine appears to be related to the peralkaline magmatic environment and low temperature (Brousse and Rançon 1984).

\subsection{Amphibole}

Amphibole phenocrysts (Tab. 4) are classified as arfvedsonite according to the nomenclature diagram of Leake et al. (1997; see Fig. 2b). High F contents of up to 2.9 wt. \% indicate crystallization under low oxygen fugacity at a late-magmatic stage (Strong and Taylor 1984). The presence of arfvedsonite is characteristic of the most highly evolved peralkaline rocks of both under- and oversaturated suites (Mitchell and Platt 1978).

\subsection{Fe-Ti oxide}

At Hadjer Hidjer ilmenite occurs as anhedral crystals with $\mathrm{FeO}$ and $\mathrm{MnO}$ contents reaching 45.9 wt. \% and 3.2 wt. $\%$, respectively (Tab. 5 ). 
Tab. 5 Ilmenite compositions (wt. $\%$ and a.p.f.u. on the basis of 6 oxygens)

\begin{tabular}{|c|c|c|c|c|c|c|c|}
\hline Sample & DH3 & DH3 & DH3 & DH3 & DH4 & DH4 & DH4 \\
\hline $\mathrm{SiO}_{2}$ (wt. \%) & 0.00 & 0.05 & 0.01 & 0.00 & 0.00 & 0.00 & 0.01 \\
\hline $\mathrm{TiO}_{2}$ & 49.98 & 50.39 & 50.78 & 51.19 & 52.74 & 51.74 & 51.89 \\
\hline $\mathrm{Al}_{2} \mathrm{O}_{3}$ & 0.01 & 0.09 & 0.04 & 0.02 & 0.00 & 0.00 & 0.00 \\
\hline $\mathrm{Cr}_{2} \mathrm{O}_{3}$ & 0.06 & 0.03 & 0.00 & 0.00 & 0.09 & 0.00 & 0.00 \\
\hline $\mathrm{FeO}$ & 45.53 & 44.34 & 43.35 & 43.77 & 43.70 & 45.89 & 44.57 \\
\hline $\mathrm{MnO}$ & 1.62 & 1.44 & 2.27 & 2.40 & 3.20 & 2.22 & 2.40 \\
\hline $\mathrm{MgO}$ & 0.10 & 0.06 & 0.02 & 0.02 & 0.00 & 0.02 & 0.03 \\
\hline $\mathrm{CaO}$ & 0.00 & 0.11 & 0.03 & 0.01 & 0.09 & 0.01 & 0.01 \\
\hline Sum & 97.30 & 96.51 & 96.50 & 97.41 & 99.82 & 99.88 & 98.91 \\
\hline $\mathrm{Fe}_{2} \mathrm{O}_{3}$ (calc.) & 2.66 & 0.74 & 0.04 & 0.23 & & 1.83 & 0.42 \\
\hline FeO (calc.) & 43.14 & 43.68 & 43.31 & 43.56 & 44.08 & 44.24 & 44.19 \\
\hline Sum (calc.) & 97.57 & 96.58 & 96.50 & 97.43 & 99.78 & 100.06 & 98.95 \\
\hline Si (a.p.f.u) & 0.000 & 0.003 & 0.001 & 0.000 & 0.000 & 0.000 & 0.001 \\
\hline $\mathrm{Ti}$ & 1.947 & 1.980 & 1.998 & 1.995 & 2.006 & 1.965 & 1.991 \\
\hline $\mathrm{Al}$ & 0.001 & 0.006 & 0.003 & 0.001 & 0.000 & 0.000 & 0.000 \\
\hline $\mathrm{Cr}$ & 0.003 & 0.001 & 0.000 & 0.000 & 0.004 & 0.000 & 0.000 \\
\hline $\mathrm{Fe}^{3+}$ & 0.104 & 0.029 & 0.002 & 0.009 & & 0.070 & 0.016 \\
\hline $\mathrm{Fe}^{2+}$ & 1.868 & 1.908 & 1.894 & 1.888 & 1.864 & 1.868 & 1.885 \\
\hline $\mathrm{Mn}$ & 0.071 & 0.064 & 0.101 & 0.105 & 0.137 & 0.095 & 0.104 \\
\hline $\mathrm{Mg}$ & 0.008 & 0.005 & 0.002 & 0.002 & 0.000 & 0.002 & 0.002 \\
\hline $\mathrm{Ca}$ & 0.000 & 0.006 & 0.002 & 0.001 & 0.005 & 0.001 & 0.001 \\
\hline $\mathrm{Fe}^{3+} / \mathrm{Fe}^{2+}$ & 0.056 & 0.015 & 0.001 & 0.005 & & 0.037 & 0.009 \\
\hline
\end{tabular}

\subsection{Alkali feldspars}

As demonstrated in the Ab-AnOr diagram (Fig. 3; see Tab. 6), there are three main groups of alkali feldspars, separated by considerable gaps: (1) nearly pure albite $\left(\mathrm{Ab}_{98-97}\right),(2)$ anorthoclaseNa-sanidine ( $\left.\mathrm{Or}_{18-44} \mathrm{Ab}_{75-52} \mathrm{An}_{0.3-7}\right)$ and (3) nearly pure sanidine $\left(\mathrm{Ab}_{98-96}\right)$.

\section{6. ${ }^{40} \mathrm{Ar}-{ }^{39} \mathrm{Ar}$ ages}

The new dating on a peralkaline rhyolite (DE) from Hadjer el Hamis yielded an age of $69.4 \pm 0.4 \mathrm{Ma}$ (electronic supplement, Appendix 1), which falls within the error of the previously determined age for peralkaline rhyolites from the same dome

Fig. 4 New age for peralkaline rhyolites from Lake Chad in context of other ages for igneous rocks from the "Cameroon Hot Line" (data from Déruelle et al. 2007 and references therein). For original data, see electronic supplement, Appendix 2.




Tab. 6 Alkali feldspar compositions (wt. \% and a.p.f.u. on the basis of 8 oxygens)

\begin{tabular}{|c|c|c|c|c|c|c|c|c|c|c|c|c|c|c|}
\hline $\begin{array}{l}\text { Sample } \\
\text { description }\end{array}$ & $\begin{array}{r}\text { DH3 } \\
\text { ph }\end{array}$ & $\begin{array}{c}\text { DH3 } \\
\text { ph }\end{array}$ & $\begin{array}{c}\text { DH4 } \\
\text { ph }\end{array}$ & $\begin{array}{c}\text { DH4 } \\
\text { ph }\end{array}$ & $\begin{array}{c}\mathrm{DH} 4 \\
\mathrm{ph}\end{array}$ & $\begin{array}{c}\mathrm{DH} 4 \\
\mathrm{ph}\end{array}$ & $\begin{array}{c}\mathrm{DH} 4 \\
\mathrm{ph}\end{array}$ & $\begin{array}{l}\mathrm{DE} \\
\mathrm{ph}\end{array}$ & $\begin{array}{c}\mathrm{DE} \\
\mathrm{ph}\end{array}$ & $\begin{array}{c}\mathrm{DE} \\
\mathrm{ph}\end{array}$ & $\begin{array}{c}\text { FB1 } \\
\text { ph }\end{array}$ & $\begin{array}{c}\text { FB1 } \\
\text { ph }\end{array}$ & $\begin{array}{c}\text { FB1 } \\
\text { ph }\end{array}$ & $\begin{array}{c}\text { FB1 } \\
\mathrm{ph}\end{array}$ \\
\hline $\mathrm{SiO}_{2}$ (wt. \%) & 67.52 & 65.84 & 67.48 & 67.23 & 67.42 & 65.65 & 67.11 & 68.30 & 63.88 & 66.26 & 68.83 & 69.13 & 64.65 & 62.58 \\
\hline $\mathrm{Al}_{2} \mathrm{O}_{3}$ & 18.68 & 20.21 & 19.12 & 18.63 & 18.82 & 17.89 & 18.72 & 18.65 & 18.35 & 18.16 & 19.60 & 19.45 & 18.41 & 14.39 \\
\hline $\mathrm{FeO}$ & 0.47 & 0.21 & 0.39 & 0.51 & 1.25 & 0.06 & 0.33 & 1.39 & 0.05 & 1.16 & 0.46 & 0.04 & 0.00 & 7.03 \\
\hline $\mathrm{CaO}$ & 0.02 & 1.47 & 0.14 & 0.03 & 0.01 & 0.11 & 0.02 & 0.06 & 0.00 & 0.03 & 0.02 & 0.03 & 0.02 & 0.09 \\
\hline $\mathrm{Na}_{2} \mathrm{O}$ & 7.74 & 8.59 & 7.73 & 8.07 & 5.65 & 0.34 & 6.99 & 11.24 & 0.27 & 6.43 & 11.55 & 11.12 & 0.13 & 1.80 \\
\hline $\mathrm{K}_{2} \mathrm{O}$ & 5.64 & 3.19 & 5.63 & 5.52 & 7.34 & 15.84 & 6.45 & 0.12 & 16.76 & 7.18 & 0.18 & 0.46 & 16.41 & 12.60 \\
\hline Sum & 100.07 & 99.51 & 100.49 & 99.99 & 100.49 & 99.89 & 99.62 & 99.76 & 99.31 & 99.22 & 100.64 & 100.23 & 99.62 & 98.49 \\
\hline $\mathrm{Si}$ (a.p.f.u) & 3.008 & 2.934 & 2.993 & 2.997 & 3.007 & 3.027 & 3.007 & 3.005 & 2.985 & 3.003 & 2.992 & 3.009 & 2.999 & 2.977 \\
\hline $\mathrm{Al}$ & 0.981 & 1.061 & 1.000 & 0.979 & 0.989 & 0.972 & 0.989 & 0.967 & 1.011 & 0.970 & 1.004 & 0.998 & 1.006 & 0.807 \\
\hline $\mathrm{Fe}^{2+}$ & 0.018 & 0.008 & 0.014 & 0.000 & 0.047 & 0.002 & 0.012 & 0.051 & 0.000 & 0.038 & 0.017 & 0.001 & 0.000 & 0.110 \\
\hline $\mathrm{Ca}$ & 0.001 & 0.070 & 0.007 & 0.001 & 0.000 & 0.005 & 0.001 & 0.003 & 0.000 & 0.001 & 0.001 & 0.001 & 0.001 & 0.005 \\
\hline $\mathrm{Na}$ & 0.670 & 0.744 & 0.667 & 0.700 & 0.490 & 0.030 & 0.609 & 0.962 & 0.025 & 0.567 & 0.976 & 0.941 & 0.012 & 0.167 \\
\hline $\mathrm{K}$ & 0.320 & 0.181 & 0.319 & 0.314 & 0.418 & 0.932 & 0.369 & 0.007 & 0.999 & 0.415 & 0.010 & 0.026 & 0.971 & 0.765 \\
\hline An (mol. \%) & 0.26 & 7.18 & 1.50 & 0.33 & 4.62 & 0.82 & 1.16 & 1.80 & 0.36 & 1.40 & 1.34 & 0.28 & 0.41 & 5.73 \\
\hline $\mathrm{Ab}$ & 67.49 & 74.65 & 66.64 & 68.82 & 51.52 & 3.13 & 61.58 & 97.53 & 2.41 & 56.93 & 97.66 & 97.11 & 1.18 & 16.85 \\
\hline Or & 32.25 & 18.17 & 31.86 & 30.85 & 43.86 & 96.05 & 37.26 & 0.67 & 97.23 & 41.67 & 1.00 & 2.61 & 98.40 & 77.42 \\
\hline
\end{tabular}

(68.9 1.4 Ma: Schroeter and Gear 1973) (Fig. 4); This suggests that all components of the dome are contemporaneous. The likely rapid cooling of the rocks together with the freshness of the sample lead us to regard the $\sim 69 \mathrm{Ma}$ age as reflecting the Late Cretaceous emplacement of the volcanic rocks in the Lake Chad area.

\section{Geochemistry}

The samples analyzed in the present study fall within the rhyolite field in TAS diagram (Fig. 5a). They have peralkaline index (P.I. $=$ molar $\left.\left[\mathrm{Na}_{2} \mathrm{O}+\mathrm{K}_{2} \mathrm{O}\right] / \mathrm{Al}_{2} \mathrm{O}_{3}\right)$ higher than unity. Using the $\mathrm{Al}_{2} \mathrm{O}_{3} v s \mathrm{FeO}_{\mathrm{t}}$ plot (Fig. 5b; Macdonald 1974), peralkaline silicic lava from Hadjer el Hamis is classified as comendite, while those from Hadjer Bigli and Hadjer Hidjer straddle the boundary to the pantellerite domain. Differentiation index (D.I. $=\sum Q t z+O r+A b+N e+L c$, CIPW norm) ranges between 89.9 and 93.4 , with low $\mathrm{mg} \#(100 \times$ molar $\mathrm{MgO} /$ $\left.\left[\mathrm{MgO}+\mathrm{FeO}_{\mathrm{t}}\right]\right)$ values (2-6). Major- and trace-element compositions for the studied samples are reported in Tab. 7.

\subsection{Major elements}

Apart from the low $\mathrm{FeO}_{\mathrm{t}}$ contents for peralkaline rhyolites from Hadjer el Hamis, the compositional trend from Hadjer Hidjer to Hadjer el Hamis, then to Hadjer Bigli is continuous and characterized by an increase of $\mathrm{SiO}_{2}$ from 68.2 to 74.5 wt. \%, accompanied by a decrease of $\mathrm{TiO}_{2}$, $\mathrm{Al}_{2} \mathrm{O}_{3}, \mathrm{CaO}, \mathrm{MgO}$ and $\mathrm{Na}_{2} \mathrm{O}$ (see Tab. 7).

\subsection{Trace elements}

Overall, the lavas from Lake Chad are characterized by a progressive enrichment in LREE, $\mathrm{Rb}, \mathrm{Zr}, \mathrm{Nb}, \mathrm{Y}$ and $\mathrm{Th}$, whereas $\mathrm{Ba}, \mathrm{Sr}, \mathrm{Co}, \mathrm{V}$ decrease from Hadjer Hidjer to Hadjer el Hamis, then to Hadjer Bigli (Tab. 7). The $\mathrm{Zr} / \mathrm{Nb}$ ratios of peralkaline rhyolites from Hadjer Bigli $(\sim 6)$ are different from those of Hadjer Hidjer and Hadjer el Hamis (just below 8). Primitive mantle-normalized (McDonough and Sun 1995) REE patterns for all samples are subparallel (Fig. 6). The main features of silicic lavas from Lake Chad, as differentiation increases from Hadjer Hidjer to Hadjer el Hamis, then to Hadjer Bigli are: (1) increase in total concentrations of REE, (2) slight increase in LREE/HREE enrichment, with $\mathrm{La}_{\mathrm{N}} / \mathrm{Yb}_{\mathrm{N}}$ ratios ranging from 8.4 to 12.6 , (3) development of a progressively deeper negative Eu anomaly. Primitive mantlenormalized (McDonough and Sun 1995) multi-element patterns (Fig. 7) show slight positive $\mathrm{Zr}$ and negative $\mathrm{Ba}$, $\mathrm{P}, \mathrm{Sr}$ and Ti anomalies. Peralkaline rhyolites from entire "Cameroon Hot Line" exhibit similar anomalies (Ngounouno et al. 1997, 2000, 2003; Marzoli et al. 1999; Vicat et al. 2002; Wandji et al. 2008). Additionally, a negative $\mathrm{K}$ anomaly characterizes the Hadjer Bigli patterns.

The silicic lavas studied form two groups: (1) peralkaline rhyolites from Hadjer Hidjer and Hadjer el Hamis show $\mathrm{La}_{\mathrm{N}} / \mathrm{Yb}_{\mathrm{N}}$ ratios relatively low, between 8.4 and 9.0, and low $\mathrm{Zr}(647-709 \mathrm{ppm})$ and $\mathrm{Nb}(84-90)$ concentrations $(\mathrm{Zr} / \mathrm{Nb}: \sim 8)$. This group (group-1) corresponds to weakly peralkaline lavas characterized by the presence of fayalite-rich olivine and in terms of $\mathrm{Zr}$ and $\mathrm{Nb}$ are less differentiated rhyolites, (2) peralkaline rhyolites 


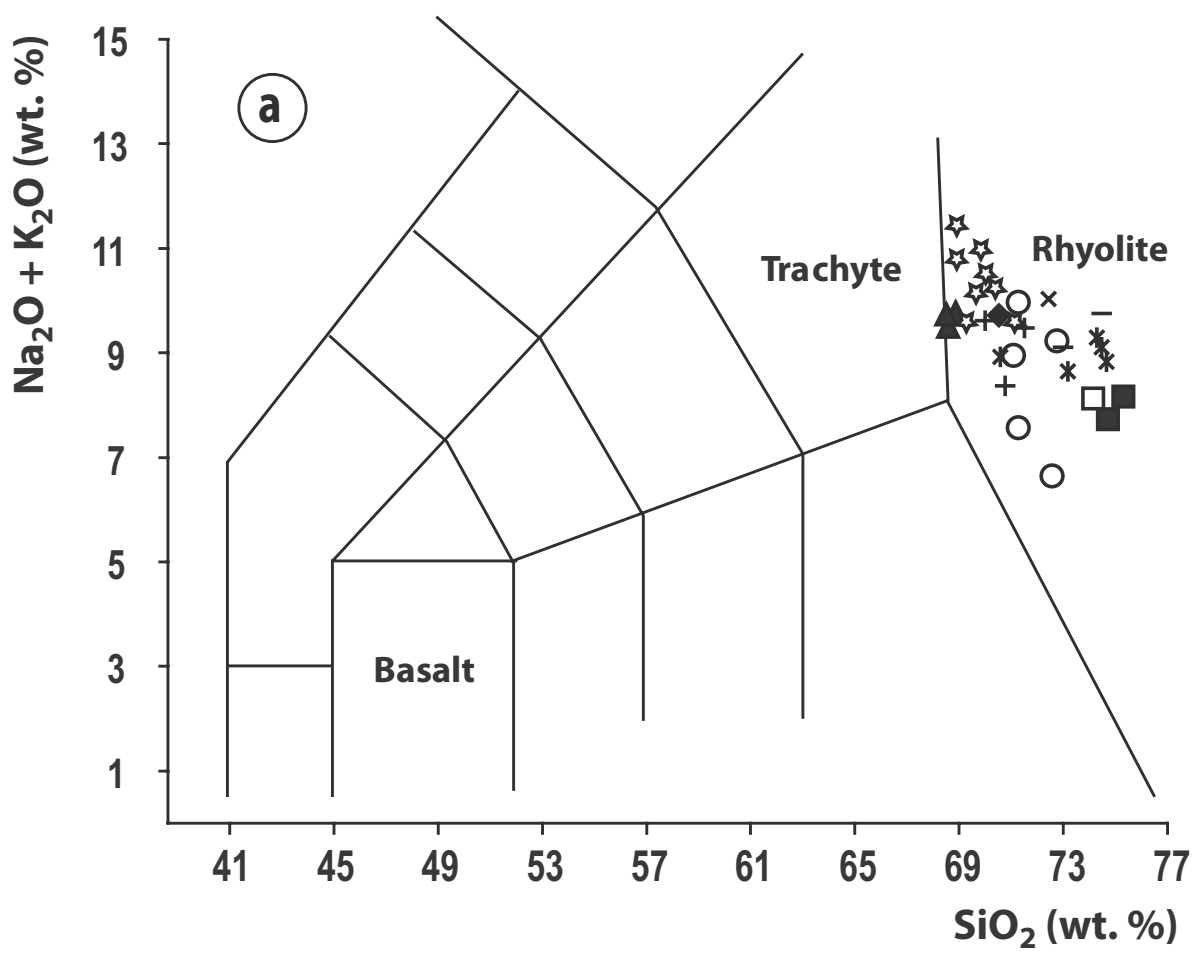

Fig. 5 Total alkali-silica (TAS) diagram (Le Bas et al. 1986) (a) and $\mathrm{FeO}_{\mathrm{t}}$ $\mathrm{Al}_{2} \mathrm{O}_{3}$ classification diagram for peralkaline rocks (Macdonald 1974) (b). Data from Ethiopia (Peccerillo et al. 2007; Ronga et al. 2010), Kenya (Scaillet and Macdonald 2003), Pantelleria Island, Italy (Avanzinelli et al. 2004), Mbépit Massif (Wandji et al. 2008) Kapsiki Plateau, Benue Valley (Ngounouno et al. 2000, 2003) and Lake Chad (Vicat et al. 2002).



from Hadjer Bigli yield normalized patterns showing a high LREE/HREE enrichment $\left(\mathrm{La}_{\mathrm{N}} / \mathrm{Yb}_{\mathrm{N}}=10.7-12.6\right)$. This group (group-2) corresponds to highly peralkaline rhyolites which are characterized by the absence of fayalite-rich olivine and by relatively high HFSE contents (High Field-Strength Elements; Zr: 1926-2445 ppm; Nb: 314-414 ppm; Zr/Nb: 6).

\section{Discussion}

\subsection{Geochronological implications}

Radiometric ages for plutonic and volcanic rocks along the "Cameroon Hot Line" span an interval between 0 Ma and 69 Ma (Fig. 4). The current work indicates that 
Tab. 7 Whole-rock major- and trace-element analyses of peralkaline rhyolites from Lake Chad

\begin{tabular}{|c|c|c|c|c|c|c|}
\hline \multirow{2}{*}{$\begin{array}{l}\text { Location } \\
\text { Sample }\end{array}$} & \multicolumn{3}{|c|}{ Hadjer Hidjer } & \multirow{2}{*}{$\begin{array}{c}\text { Hadjer el Hamis } \\
\text { DE }\end{array}$} & \multicolumn{2}{|c|}{ Hadjer Bigli } \\
\hline & DH2 & DH3 & DH4 & & FB1 & FB3 \\
\hline GPS & $14^{\circ} 39^{\prime} \mathrm{E}$ & $14^{\circ} 39^{\prime} \mathrm{E}$ & $14^{\circ} 39^{\prime} \mathrm{E}$ & $14^{\circ} 51^{\prime} \mathrm{E}$ & $14^{\circ} 40^{\prime} \mathrm{E}$ & $14^{\circ} 40^{\prime} \mathrm{E}$ \\
\hline coordinates & $12^{\circ} 47^{\prime} \mathrm{N}$ & $12^{\circ} 47^{\prime} \mathrm{N}$ & $12^{\circ} 47^{\prime} \mathrm{N}$ & $12^{\circ} 52^{\prime} \mathrm{N}$ & $12^{\circ} 48^{\prime} \mathrm{N}$ & $12^{\circ} 48^{\prime} \mathrm{N}$ \\
\hline $\mathrm{SiO}_{2}$ (wt. \%) & 68.65 & 68.18 & 68.15 & 70.54 & 75.31 & 74.58 \\
\hline $\mathrm{TiO}_{2}$ & 0.41 & 0.43 & 0.42 & 0.29 & 0.18 & 0.19 \\
\hline $\mathrm{Al}_{2} \mathrm{O}_{3}$ & 12.21 & 12.47 & 12.42 & 12.06 & 9.72 & 9.51 \\
\hline $\mathrm{FeO}_{\mathrm{t}}$ & 6.04 & 6.33 & 6.13 & 4.83 & 5.80 & 5.40 \\
\hline $\mathrm{MnO}$ & 0.18 & 0.18 & 0.19 & 0.22 & 0.08 & 0.06 \\
\hline $\mathrm{MgO}$ & 0.10 & 0.10 & 0.12 & 0.04 & 0.03 & 0.04 \\
\hline $\mathrm{CaO}$ & 1.04 & 1.01 & 1.11 & 0.31 & 0.27 & 0.24 \\
\hline $\mathrm{Na}_{2} \mathrm{O}$ & 5.34 & 5.36 & 5.32 & 5.00 & 3.82 & 3.28 \\
\hline $\mathrm{K}_{2} \mathrm{O}$ & 4.35 & 4.37 & 4.30 & 4.71 & 4.37 & 4.47 \\
\hline $\mathrm{P}_{2} \mathrm{O}_{5}$ & 0.06 & 0.06 & 0.06 & 0.10 & $<$ D.L. & $<$ D.L. \\
\hline L.O.I. & 0.31 & 0.44 & 0.30 & 0.74 & 1.31 & 1.71 \\
\hline Sum & 98.69 & 98.91 & 98.50 & 98.82 & 100.88 & 99.48 \\
\hline D.I. & 89.90 & 89.20 & 89.03 & 93.38 & 92.38 & 92.01 \\
\hline P.I. & 1.11 & 1.09 & 1.08 & 1.10 & 1.13 & 1.08 \\
\hline$\underline{\mathrm{mg} \#}$ & 0.05 & 0.05 & 0.06 & 0.02 & 0.01 & 0.02 \\
\hline$\overline{\mathrm{Be}}(\mathrm{ppm})$ & 4.27 & 4.06 & 4.38 & 4.14 & 15.50 & 10.10 \\
\hline $\mathrm{Rb}$ & 90 & 92 & 85 & 72 & 247 & 278 \\
\hline $\mathrm{Sr}$ & 67 & 72 & 82 & 16 & 4 & 5 \\
\hline Cs & 0.76 & 0.67 & 0.58 & 0.38 & 0.51 & 0.83 \\
\hline $\mathrm{Ba}$ & 417 & 523 & 506 & 260 & 9 & 10 \\
\hline $\mathrm{V}$ & 1.2 & 1.5 & 1.4 & $<$ D.L. & $<$ D.L. & $<$ D.L. \\
\hline $\mathrm{Cr}$ & $<$ D.L. & $<$ D.L. & $<$ D.L. & $<$ D.L. & $<$ D.L. & $<$ D.L. \\
\hline Co & 0.6 & 0.8 & 0.6 & < D.L. & $<$ D.L. & $<$ D.L. \\
\hline $\mathrm{Ni}$ & $<$ D.L. & $<$ D.L. & $<$ D.L. & $<$ D.L. & $<$ D.L. & $<$ D.L. \\
\hline $\mathrm{Cu}$ & $<$ D.L. & $<$ D.L. & $<$ D.L. & $<$ D.L. & $<$ D.L. & $<$ D.L. \\
\hline $\mathrm{Zn}$ & 196.9 & 207.7 & 196.5 & 219.7 & 538.9 & 522.5 \\
\hline $\mathrm{Y}$ & 56.9 & 62.8 & 57.3 & 66.5 & 244.0 & 222.7 \\
\hline $\mathrm{Zr}$ & 647 & 655 & 680 & 709 & 2445 & 1926 \\
\hline $\mathrm{Nb}$ & 84.9 & 86.1 & 87.4 & 89.9 & 414.2 & 314.3 \\
\hline $\mathrm{Hf}$ & 14.60 & 14.59 & 15.09 & 15.00 & 61.97 & 47.95 \\
\hline $\mathrm{Ta}$ & 6.35 & 6.46 & 6.30 & 6.53 & 28.40 & 21.11 \\
\hline $\mathrm{Th}$ & 10.5 & 10.8 & 10.8 & 9.5 & 47.0 & 43.9 \\
\hline $\mathrm{U}$ & 2.89 & 2.91 & 2.93 & 2.76 & 11.27 & 12.42 \\
\hline $\mathrm{Pb}$ & 10.7 & 18.0 & 15.9 & 10.4 & 57.3 & 23.9 \\
\hline $\mathrm{Ga}$ & 38.5 & 39.2 & 39.4 & 40.6 & 48.8 & 41.8 \\
\hline $\mathrm{La}$ & 69.7 & 73.2 & 69.9 & 74.0 & 393.5 & 268.4 \\
\hline $\mathrm{Ce}$ & 148 & 156 & 152 & 167 & 599 & 503 \\
\hline $\operatorname{Pr}$ & 16.8 & 17.5 & 17.2 & 18.4 & 94.3 & 57.8 \\
\hline $\mathrm{Nd}$ & 67.8 & 71.8 & 69.7 & 74.4 & 330.4 & 209.8 \\
\hline $\mathrm{Sm}$ & 14.00 & 14.90 & 14.42 & 15.65 & 62.74 & 41.75 \\
\hline $\mathrm{Eu}$ & 2.67 & 3.02 & 2.89 & 2.87 & 5.64 & 3.08 \\
\hline $\mathrm{Gd}$ & 12.33 & 13.60 & 12.82 & 14.03 & 49.10 & 37.92 \\
\hline $\mathrm{Tb}$ & 1.95 & 2.11 & 1.96 & 2.25 & 7.50 & 6.20 \\
\hline Dy & 11.27 & 12.24 & 11.21 & 13.03 & 42.25 & 36.26 \\
\hline Ho & 2.12 & 2.28 & 2.08 & 2.40 & 7.95 & 6.87 \\
\hline $\mathrm{Er}$ & 5.79 & 6.23 & 5.58 & 6.52 & 22.44 & 18.81 \\
\hline $\mathrm{Tm}$ & 0.836 & 0.905 & 0.799 & 0.930 & 3.300 & 2.671 \\
\hline $\mathrm{Yb}$ & 5.52 & 5.88 & 5.27 & 5.99 & 21.30 & 17.10 \\
\hline $\mathrm{Lu}$ & 0.838 & 0.888 & 0.797 & 0.911 & 3.058 & 2.457 \\
\hline $\mathrm{Eu} / \mathrm{Eu}^{*}$ & 0.65 & 0.65 & 0.62 & 0.59 & 0.24 & 0.31 \\
\hline $\mathrm{Zr} / \mathrm{Nb}$ & 7.6 & 7.6 & 7.8 & 7.9 & 5.9 & 6.1 \\
\hline $\mathrm{La}_{\mathrm{N}} / \mathrm{Yb}_{\mathrm{N}}$ & 8.58 & 8.48 & 9.02 & 8.41 & 12.57 & 10.68 \\
\hline$\sum$ REE & 360 & 381 & 367 & 398 & 1642 & 1212 \\
\hline
\end{tabular}

FeOt: total $\mathrm{Fe}_{\text {as }} \mathrm{Fe}^{3+}$; L.O.I.: Loss on ignition; D.I.: Differentiation index;

P.I.: Peralkaline index; D.L.: detection limit 


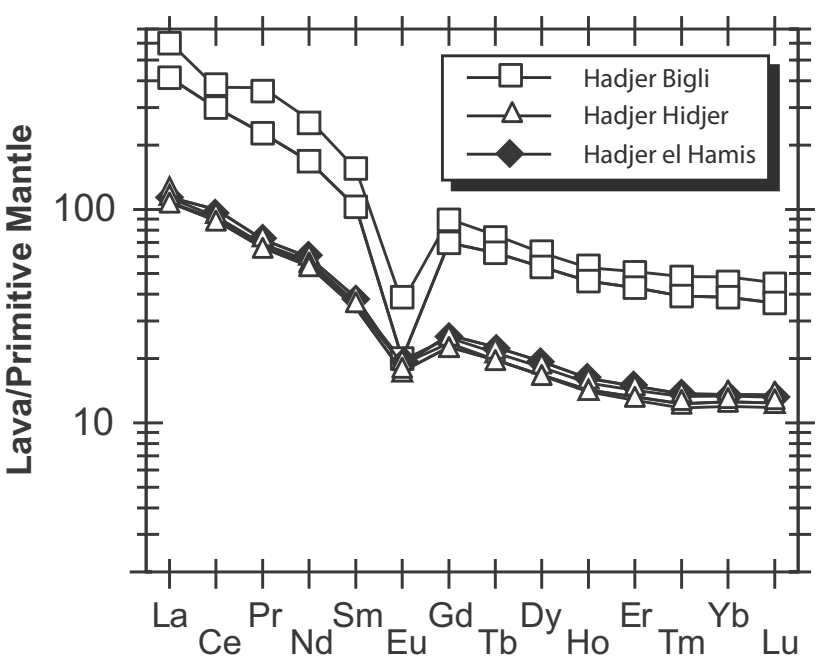

Fig. 6 Representative primitive mantle-normalized (McDonough and Sun 1995) REE diagrams for peralkaline rhyolites from Lake Chad.



$\mathrm{Rb}$ Th Nb K Ce Pb Nd Sm Zr Eu Tb Y Lu

Fig. 7 Representative primitive mantle-normalized (McDonough and Sun 1995) multi-element diagrams for peralkaline rhyolites from Lake Chad.

the volcanic eruptions started at northeastern end of the Line, in the Lake Chad Basin, in Late Cretaceous ( $\sim 69$ Ma). The rhyolites from Mbépit Massif are much younger $(45.5 \pm 1.1 \mathrm{Ma}$ : Wandji et al. 2008; see Tab. 1$)$; the volcanic manifestations were thus absent till c. $45 \mathrm{Ma}$, even though minor plutonic complexes were emplaced during this period. Both plutonic and volcanic activity took place between $65 \mathrm{Ma}$ and $30 \mathrm{Ma}$, recorded between Kapsiki and Bambouto at latitudes of $5-11^{\circ} \mathrm{N}$. Volcanic eruptions were clearly predominant along the whole Cameroon Line between $10^{\circ} \mathrm{N}$ and $1.5^{\circ} \mathrm{S}$, from about $25 \mathrm{Ma}$ to recent, whereas minor plutonic intrusions are recorded during this time span at Mts. Rumpi. The most recent eruptions have been witnessed at Bioko and Mount Cameroon, respectively, in 1923 and 2000.
The absence of a progressive age distribution (Fig. 4), which would be expected in the case of a plume (Fitton and Dunlop 1985), lead us to refute the hypothesis of a hot spot for the origin of the "Cameroon Hot Line", which was previously considered as a Cenozoic to present tectonomagmatic zone (Déruelle et al. 2007; Nkouandou and Temdjim 2011). The situation must be reconsidered on the basis of the new dating of the Lake Chad volcanic rocks.

\subsection{Inferences concerning conditions of crystallization}

The crystallization of Na-rich clinopyroxene (i.e., aegirine-augite and aegirine) and other Na-bearing phases, such as arfvedsonite, contribute efficaciously to the formation of peralkaline magmas (White et al. 2005). The stability and composition of clinopyroxene and arfvedsonite are temperature-, $\mathrm{H}_{2} \mathrm{O}$ activity-, melt composition- and $f \mathrm{O}_{2}$-dependent (Scaillet and Macdonald 2001). Anhydrous conditions inhibit aegirine and Narich amphibole crystallization; also low $f \mathrm{O}_{2}$ destabilizes Na-rich clinopyroxene that transforms into a fayalite-ilmenite (Ronga et al. 2010). The late destabilization of arfvedsonite in the rhyolitic liquids of Lake Chad took place at low temperatures $\left(<900^{\circ} \mathrm{C}\right)$, as evidenced by the Ca-rich composition of fayalite. Low $f_{2}$ values (below QFM buffer) at temperatures less than $800{ }^{\circ} \mathrm{C}$ favour the development of peralkaline liquids without the appearance of aegirine or sodic amphibole as liquidus phases (Ronga et al. 2010). The destabilization of arfvedsonite phenocrysts (breaking down into fayalite and ilmenite) was probably caused by a sudden increase in $f_{2}$ of peralkaline silicic magmas, which promoted the enrichment of $\mathrm{Na}$ in clinopyroxene and the crystallization of aegirine (Njonfang and Nono 2003). Similar effect was shown also for the aegirine from Djinga Tadorgal and São Tomé phonolites (Mbowou 2009). The occurrence of alkali feldspars with intermediate compositions (anorthoclase-Na-sanidine) provides an evidence for hypersolvus crystallization conditions (Wandji et al. 2008). Nearly pure albite and sanidine are regarded as reflecting alkali exchange during sub-solidus hydrothermal reactions or as corresponding to late-stage recrystallization at hydrothermal conditions. The resorption rims (corrosion gulfs), seen on quartz phenocrysts in Hadjer el Hamis rhyolitic lavas, indicate a disequilibrium with the melt. They were probably generated during decompression of the rhyolitic magmas, during ascent to the surface upon eruption. Furthermore, given the quartz stability mainly in the low-temperature magma, thermal erosion and fluid-melt infiltration could have also contributed to the occurrence of resorbed quartz. 


\subsection{Implications of various distributions of geochemical data}

Silicic lavas from Lake Chad (pantellerites and comendites) may be genetically related to each other, corresponding to a series of progressively differentiated magmas. Progressive depletion in $\mathrm{FeO}_{\mathrm{t}}, \mathrm{MgO}, \mathrm{CaO}$ and Co from group-1 to group-2 peralkaline rhyolites indicates a simultaneous fractional crystallization of mafic minerals (Fo-rich olivine, and/or clinopyroxene) from a basaltic parental magma. The well-developed negative K, $\mathrm{Ba}, \mathrm{Ti}, \mathrm{Sr}$ and $\mathrm{Eu}$ anomalies of the peralkaline rhyolites are consistent with crystallization of arfvedsonite $(\mathrm{K}, \mathrm{Ti})$, ilmenite (Ti) and alkali feldspar (K, Ba, Sr, Eu). Furthermore, the development of progressively deeper negative $\mathrm{Eu}$ anomaly with $\mathrm{Eu} / \mathrm{Eu}^{*}\left(\mathrm{Eu} / \mathrm{Eu}^{*}=\mathrm{Eu}_{\mathrm{N}} / \sqrt{ }\left(\mathrm{Sm}_{\mathrm{N}} \times \mathrm{Gd}_{\mathrm{N}}\right)\right)$ ranging from $0.24-0.31$ in Hadjer Bigli to $0.59-0.65$ in Hadjer Hidjer and Hadjer el Hamis, can be explained by major feldspar fractionation. The crystallization of $\mathrm{Na}-\mathrm{K}-$ rich minerals (e.g., fluoro-arfvedsonite, aegirine-augite, aegirine, alkali feldspars), which fractionate alkalis, contributes efficiently to the $\mathrm{SiO}_{2}$-oversaturation of residual silicic magma and likely leads to the crystallization of quartz. Positive $\mathrm{Zr}$ anomalies are linked to the inhibition of the zircon crystallization (Watson 1979) or to the increase of the $\mathrm{Fe}^{3+} / \mathrm{Fe}^{\text {total }}$ ratios during the late stages of magmatic evolution (Duggan 1988; Farges et al. 1994). The HFSE (e.g., Ti, Zr, HREE, $\mathrm{Y}$ and $\mathrm{Nb}$ ) are usually not transported in aqueous fluids, except when these contain high proportion of certain complexing agents such as $\mathrm{F}^{-}$. As shown by Pearce and Norry (1979), F-rich arfvedsonite and F-rich fluids control $\mathrm{Zr}$ distribution. Furthermore, the observed incompatible elements enrichment (e.g., $\mathrm{Rb}, \mathrm{Zr}, \mathrm{Nb}, \mathrm{Th}, \mathrm{U}$ and LREE) is consistent with a formation from a highly fractionated residual liquid.

For comparison, peralkaline rhyolites of Kapsiki Plateau enclosing small xenoliths of basalt and containing ilmenite, arfvedsonite, aegirine-augite phenocrysts, have high concentrations of $\mathrm{Zr}$ (up to $2180 \mathrm{ppm}$ ) and $\mathrm{Nb}$ (up to $780 \mathrm{ppm}$ ) with relatively high $\mathrm{Zr} / \mathrm{Nb}$ ratios (6.3-8.3) similar to those of Lake Chad rhyolites. Conversely, Benue Valley rhyolites are characterized by a sodic mineralogy (aegirine-augite, richterite and arfvedsonite) with lower $\mathrm{Zr} / \mathrm{Nb}$ ratios $(\sim 1.7)$, possibly due to aegirine-augite (up to 1.07 wt. $\% \mathrm{ZrO}_{2}$ ) fractionation (Ngounouno et al. 2003). Mbépit Massif contains phenocrysts of $\mathrm{Na}-\mathrm{K}$-feldspar, quartz and $\mathrm{Fe}-\mathrm{Ti}$ oxides with higher $\mathrm{Zr} / \mathrm{Nb}$ ratios (10-11). This indicates that rhyolites similar to those of Lake Chad are scarce within the "Cameroon Hot Line".

\subsection{Genesis of silicic magmas}

Contrasting interpretations are proposed to explain the origin of continental-rift peralkaline rhyolites (see Ronga et al. 2010 and references therein): (1) fractional crystallization of a basaltic parental melt slightly contaminated by crust (Peccerillo et al. 2003, 2007) and (2) partial melting of local lower crust triggered by alkali-bearing volatiles influx (Scaillet and Macdonald 2001 and references therein) and/or basic magma underplating.

Fractional crystallization from basalts to silicic lavas would yield some geochemical characteristics of peralkaline melts but it fails to explain the lack of rocks with intermediate compositions and the high volume of evolved rocks (Peccerillo et al. 2003). However, the lack of intermediate lavas around the Lake Chad can be related to their high viscosity, preventing them from erupting. Pantellerites and comendites have higher viscosities still but they are associated to much lower density (Ronga et al. 2010).

The peralkaline rhyolites from Lake Chad could not be solely the product of fractional crystallization of basaltic melts. Some other processes, such as the transfer of F-rich fluids, could have greatly modified the composition of residual magma. These fluids, whose presence is attested by the occurrence of F-rich minerals (e.g., fluoro-arfvedsonite), could come from a metasomatised mantle source (Dautria et al. 1992; Hauri et al. 1993; McDonough and Rudnick 1998) as suggested for the lithospheric mantle beneath the "Cameroon Hot Line" (Déruelle et al. 2007). However, according to Bohrson and Reid (1997), the peralkaline silicic magmas could be generated in the lower crust, by partial melting of either old crust or young underplated basalts triggered by alkali-bearing volatiles influx. Thus, the occurrence of F-rich minerals could also be linked to such a partial melting of local, fluids-rich lower crust.

\section{Conclusions}

Petrographic features as well as major- and trace-element whole-rock geochemical data for silicic volcanic rocks from Lake Chad allow us to establish that the silicic volcanic rocks are the products of prolonged fractional crystallization of parental alkali basalts, accompanied by an influx of F-rich fluids suggested by the presence of hydrated F-rich mineral phases (fluoro-arfvedsonite) in these rocks. The fluids came presumably from a metasomatized mantle source. Peralkaline rhyolites from Lake Chad, with an age of $69.4 \pm 0.4 \mathrm{Ma}\left({ }^{40} \mathrm{Ar} /{ }^{39} \mathrm{Ar}\right.$ laser single grain sanidine dating) are likely the first erupted lavas along the "Cameroon Hot Line".

Acknowledgements. The French 'Ministère de la Coopération' is acknowledged for providing a grant to G.I.B.M. for nine-month stay in France in the 'Laboratoire de magmatologie et de géochimie inorganique et expérimentale, Université Pierre-et-Marie-Curie', Paris. F. Stoppa and 
an anonymous reviewer are thanked for their positive reviews. V. Kachlík and V. Janoušek are acknowledged for their editorial work, which improved greatly the quality of this manuscript.

Electronic supplementary material. The table of $\mathrm{Ar}-\mathrm{Ar}$ data on sanidine and overview of previous ages for volcanic rocks along the "Cameroon Hot Line" is available online at the Journal web site (http://dx.doi.org/10.3190/ jgeosci.118)

\section{References}

Avanzinelli R, Bindi L, Menchetti S, Conticelli S (2004) Crystallisation and genesis of peralkaline magmas from Pantelleria Volcano, Italy: an integrated petrological and crystal-chemical study. Lithos 73: 41-69

Baker BH, Henage LF (1977) Compositional changes during crystallization of some peralkaline silicic lavas of the Kenya Rift Valley. J Volcanol Geotherm Res 2: 17-28

Barbeau J (1956) Notice explicative sur la feuille FortLamy. Carte géologique de reconnaissance à l'échelle de 1/1000000. Direction des Mines et Géologie de l'Afrique Equatoriale Française, pp 1-35

BenkHelil J (1986) Structure et évolution du bassin intercontinental de la Bénoué (Nigeria). Unpublished Ph.D. thesis, University of Nice, pp 1-273

Bonatti E, Harrison CGA (1976) Hot lines in the Earth's mantle. Nature 263: 402-404

Bohrson WA, ReID MR (1997) Genesis of silicic peralkaline volcanic rocks in an ocean island setting by crustal melting and open-system processes: Socorro Island, Mexico. J Petrol 38: 1137-1166

Brousse R, Rançon JPh (1984) Crystallization trends of pyroxenes from agpaitic phonolites (Cantal, France). Mineral Mag 48: 39-45

Carignan J, Hild P, Mévelle G, Morel J, Yeghicheyan D (2001) Routine analyses of trace elements in geological samples using flow injection and low pressure on-line liquid chromatography couples to ICPMS: a study of geochemical reference materials BR. DR-N, UB-N, AN-G and GH. Geostand Newslett 25: 187-198

Civetta L, D’antonio M, Orsi G, Tilton GR (1998) The geochemistry of volcanic rocks from Pantelleria Island, Sicily Channel: petrogenesis and characteristics of the mantle source region. J Petrol 39: 1453-1491

Dautria JM, Dupuy C, Takherist D, Dostal J (1992) Carbonate metasomatism in the lithospheric mantle: peridotitic xenoliths from a melilititic district of the Sahara Basin. Contrib Mineral Petrol 111: 37-52

Déruelle B, Moreau C, Nkoumbou C, Kambou R, Lissom J, Nuongfang E, Ghogomu RT, Nono A (1991) The Cameroon Line: a review. In: Kampunzu AB, Lubala RT (eds) Magmatism in Extensional Structural Settings, The Phanerozoic African Plate. Springer-Verlag, Heidelberg, pp 274-327

Déruelle B, Ngounouno I, Demaiffe D (2007) The 'Cameroon Hot Line' (CHL): a unique example of active alkaline intraplate structure in both oceanic and continental lithospheres. C R Geosci 339: 589-600

Duggan MB (1988) Zirconium-rich sodic pyroxenes in felsic volcanics from the Warrumbungle Volcano, Central New South Wales, Australia. Mineral Mag 52: 491-496

DunLoP HM (1983) Strontium isotope geochemistry and potassium argon studies on volcanic rocks from the Cameroon Line, West Africa. Unpublished Ph.D. thesis, University of Edinburgh, pp 1-347

Farges F, Brown JR. GE, Calas G, Galoisy L, Waychunas GA (1994) Structural transformation in Ni-bearing Na${ }_{2} \mathrm{Si}_{2} \mathrm{O}_{5}$ glass and melt. Geophys Res Lett 21: 1931-1934

FERGUSON AK (1977) The natural occurrence of the aegirineneptunite solid solution. Contrib Mineral Petrol 60: 247-253

FitTon JG, Dunlop HM (1985) The Cameroon Line, West Africa, and its bearing on the origin of the oceanic and continental alkali basalt. Earth Planet Sci Lett 72: 23-38

Ganwa AA, Doumnang MJC, Lagmet C (2009) Pétrographie et données structurales sur les dômes rhyolitiques du sud du Lac Tchad (Dandi-Hadjer El Hamis) : relation avec la Ligne du Cameroun. Rev Cames 08: 80-85

Hauri EH, Shimizu N, Dieu JJ, Hart SR (1993) Evidence for hotspot-related carbonatite metasomatism in the oceanic upper mantle. Nature 365: 221-227

Heumann A, Davies GR (2002) U-Th disequilibrium and $\mathrm{Rb}-\mathrm{Sr}$ age constraints on the magmatic evolution of peralkaline rhyolites from Kenya. J Petrol 43: 557-577

Le Bas MJ, Le Maitre RW, Streckeisen A, Zanettin B (1986) A chemical classification of volcanic rocks based on the total alkali-silica diagram. J Petrol 27: 745-750

Leake BE, Wooley AR, Arps CES, Birch WD, Gilbert MC, Grice JD, Hawthorne F C, Kato A, Kisch HJ, KrivoviCheV VG, Linthout K, Laird J, Mandarino J, Maresch WV, Nickel EH, Rock N MS, Schumacher JC, Sмith JC, Stephenson NCN, Whittaker EJW, Youzhi G (1997) Nomenclature of amphiboles: report of the Subcommittee on Amphiboles of the International Mineralogical Association Commission on new minerals and mineral names. Mineral Mag 61: 295-321

Louis P (1970) Contribution géophysique à la connaissance géologique du bassin du Lac Tchad. Mémoire ORSTOM, 42: pp 1-311

Lowenstern JB, Mahood GA (1991) New data on magmatic $\mathrm{H}_{2} \mathrm{O}$ contents of pantellerites, with implications for petrogenesis and eruptive dynamics at Pantelleria. Bull Volcanol 54: 78-83

Macdonald R (1974) Nomenclature and petrochemistry of the peralkaline oversaturated extrusive rocks. Bull Volcanol 38: 498-516 
Macdonald R, Bagiński B (2009) The central Kenya peralkaline province: a unique assemblage of magmatic systems. Mineral Mag 73: 1-16

Marshall AS, Macdonald R, Rogers NW, Fitton JG, Tindle AG, Nejbert K, Hinton RW (2009) Fractionation of peralkaline silicic magmas: the Greater Olkaria Volcanic Complex, Kenya Rift Valley. J Petrol 50: 323-359

Marzoli A, Renne PR, Piccirillo EM, Francesca C, BelLieni G, Melfi AJ, Nyobe JB, N'ni J (1999) Silicic magmas from the continental Cameroon Volcanic Line (Oku, Bambouto and Ngaoundere): ${ }^{40} \mathrm{Ar}-{ }^{39} \mathrm{Ar}$ dates, petrology, $\mathrm{Sr}-\mathrm{Nd}-\mathrm{O}$ isotopes and their petrogenetic significance. Contrib Mineral Petrol 135: 133-150

Mbowou GIB (2009) Pétrologie du Massif de Djinga Tadorgal (Adamaoua, Cameroun): comparaison avec le volcanisme des îles de São Tomé et Príncipe et du lac Tchad ("Ligne Chaude du Cameroun"). Unpublished Ph.D. thesis, University Pierre-and-Marie Curie, Paris 6, pp 1-265

Mbowou GIB, Ngounouno I, Déruelle B (2010) Pétrologie du volcanisme bimodal du Djinga Tadorgal (Adamaoua, Cameroun). Rev Cames 11: 36-42

McDonough WF, Rudnick RL (1998) Mineralogy and composition of the upper mantle. In: Hemly RJ (ed) Ultrahigh-Pressure Mineralogy: Physics and Chemistry of the Earth's Deep Interior. Mineralogical Society of America Reviews in Mineralogy 37: 139-175

McDonough WF, Sun S-s (1995) The composition of the Earth. Chem Geol 120: 223-253

Mitchell RH, Platt RG (1978) Mafic mineralogy of ferroaugite syenite from the Coldwell alkaline complex, Ontario, Canada. J Petrol 19: 627-651

Moreau C, Regnoult J-M, Déruelle B, Robineau B (1987) A new tectonic model for the Cameroon Line, Central Africa. Tectonophysics 139: 317-334

Morimoto N (1989) Nomenclature of pyroxenes. Canad Mineral 27: 143-156

Morra V, Secchi FA, Assorgia A (1994) Petrogenetic significance of peralkaline rocks from Cenozoic calcalkaline volcanism from SW Sardinia, Italy. Chem Geol 118: 109-142

Ngounouno I, Déruelle B, Demaiffe D, Montigny R (1997) Données nouvelles sur le volcanisme cénozoïque du fossé de Garoua (Nord du Cameroun). C R Acad Sci Paris 325: 87-94

Ngounouno I, Déruelle B, Demaiffe D (2000) Petrology of the bimodal Cenozoic volcanism of the Kapsiki Plateau (northernmost Cameroon, Central Africa). J Volcanol Geotherm Res 102: 21-44

Ngounouno I, Déruelle B, Demaiffe D, Montigny R (2003) Petrology of the Cenozoic volcanism in the Upper Benue Valley, northern Cameroon (Central Africa). Contrib Mineral Petrol 145: 87-106
Nuonfang E, Nono A (2003) Clinopyroxene from some felsic alkaline rocks of the Cameroon Line, central Africa: petrological implications. Eur J Mineral 15: 527-542

Nkouandou OF, Temdjim R (2011) Petrology of spinel lherzolite xenoliths and host basaltic lava from Ngao Voglar volcano, Adamawa Massif (Cameroon Volcanic Line, West Africa): equilibrium conditions and mantle characteristics. J Geosci 56: 375-387

Nomade S, Renne PR, Vogel N, Deino AL, Sharp WD, Becker TA, Jaouni AR, Mundil R (2005) Alder Creek Sanidine (ACs-2): a Quaternary ${ }^{40} \mathrm{Ar} /{ }^{39} \mathrm{Ar}$ standard. Chem Geol 218: 319-342

Nomade S, Gauthier A, Guillou H, Pastre JF (2010) ${ }^{40} \mathrm{Ar} /{ }^{39} \mathrm{Ar}$ temporal framework for the Alleret maar lacustrine sequence (French Massif Central): volcanological and paleoclimatic implications. Quater Geochronol 5: 20-27

Pearce JA, Norry MJ (1979) Petrogenetic implications of $\mathrm{Ti}, \mathrm{Zr}, \mathrm{Y}$, and $\mathrm{Nb}$ variations in volcanic rocks. Contrib Mineral Petrol 69: 33-47

Peccerillo A, Barberio Mr, Yirgu G, Ayalew D, Barbieri M, Wu TW (2003) Relationships between mafic and peralkaline silicic magmatism in continental rift settings: a petrological, geochemical and isotopic study of the Gedemsa Volcano, Central Ethiopian Rift. J Petrol 44: 2003-2032

Peccerillo A, Donati C, Santo AP, Orlando A, Yirgu G, Ayalew D (2007) Petrogenesis of silicic peralkaline rocks in the Ethiopian Rift: geochemical evidence and volcanological implications. J Afr Earth Sci 48: 161-173

Pouchou JL, Pichoir F (1991) Quantitative analysis of homogeneous or stratified microvolumes applying the model «PAP». In: HeInRICH KFJ, Newbury DE (eds) Electron Probe Quantification. Plenum Press, New York, pp 31-75

Roeder PL, Emsle RF (1970) Olivine-liquid equilibrium. Contrib Mineral Petrol 29: 275-289

Ronga F, Lustrino M, Marzoli A, Melluso L (2010) Petrogenesis of a basalt-comendite-pantellerite rock suite: the Boseti Volcanic Complex (Main Ethiopian Rift). Mineral Petrol 98: 227-243

Scaillet B, Macdonald R (2001) Phase relations of peralkaline silicic magmas and petrogenetic implications. J Petrol 42: 825-845

SCAILlET B, MACDONALD R (2003) Experimental constraints on the relationships between peralkaline rhyolites of the Kenya Rift Valley. J Petrol 44: 1867-1894

Schroeter P, Gear D (1973) Étude des ressources en eau du bassin du lac Tchad en vue d'un programme de développement. CBLT. Ressources en eaux souterraines dans le bassin du lac Tchad. T1. Étude hydrogéologique, Rapport PNUD/FAO, Rome, Italy, pp 1-47

Simkin T, Smith JV (1970) Minor-element distribution in olivine. J Geol 78: 304-325 
Steiger RH, JÄGer E (1977) Subcommission on Geochronology: convention of the use of decay constants in geo- and cosmochronology. Earth Planet Sci Lett 36: 359-362

Strong DF, TAYlor RP (1984) Magmatic-subsolidus and oxidation trends in composition of amphiboles from silica-saturated peralkaline igneous rocks. Tschermaks Mineral Petrogr Mitt 32: 211-222

Tindle AG, WebB PC (1994) PROBE-AMPH - a spreadsheet program to classify microprobe-derived amphibole analyses. Comput Geosci 20: 1201-1228

Vicat J-P, Pouclet A, Bellion Y, Doumnang J-C (2002) Les rhyolites hyperalcalines (pantellérites) du lac Tchad. Composition et signification tectonomagmatique. C R Geosci 334: 886-891
Wandi P, Seuwui DT, Bardintzeff J-M, Bellon H, Platevoet B (2008) Rhyolites of the Mbépit Massif in the Cameroon Volcanic Line: an early extrusive volcanic episode of Eocene age. Mineral Petrol 94: 271-286

WATson EB (1979) Zircon saturation in felsic liquids: experimental result and applications to trace element geochemistry. Contrib Mineral Petrol 70: 407-419

Weaver SD, Sceal JSC, Gibson IL (1972) Trace element data relevant to the origin of the trachytic and pantelleritic lavas in the East African Rift System. Contrib Mineral Petrol 36: 181-194

White JC, Ren M, PArker DF (2005) Variation in mineralogy, temperature, and oxygen fugacity in a suite of strongly peralkaline lavas and tuffs, Pantelleria, Italy. Canad Mineral 43: 1331-1347 